\title{
Des transparents et des écrans qui communiquent en bleu méditerranée
}

Pour une rhétorique consciente et une ergonomie raisonnée des transparents et écrans d'EAO

\section{Alain Ginet}

\section{OpenEdition}

\section{Journals}

Édition électronique

URL : http://journals.openedition.org/asp/4156

DOI : $10.4000 /$ asp. 4156

ISSN : 2108-6354

\section{Éditeur}

Groupe d'étude et de recherche en anglais de spécialité

Édition imprimée

Date de publication : 1 février 1994

Pagination : 1-11

ISSN : 1246-8185

Référence électronique

Alain Ginet, "Des transparents et des écrans qui communiquent en bleu méditerranée », ASp [En ligne], 3 | 1994, mis en ligne le 21 janvier 2014, consulté le 04 mai 2019. URL : http://

journals.openedition.org/asp/4156; DOI : 10.4000/asp.4156

Ce document a été généré automatiquement le 4 mai 2019.

Tous droits réservés 


\section{Des transparents et des écrans qui communiquent en bleu méditerranée}

Pour une rhétorique consciente et une ergonomie raisonnée des transparents et écrans d'EAO

\section{Alain Ginet}

1 Voilà un titre bien peu sérieux, suggéré par la proximité de la mer et le thème du XXXIII congrès de la SAES. Ce titre avance cependant pour sa défense un espoir de pertinence par rapport au plaidoyer présenté ici pour une meilleure communication visuelle tant dans les transparents utilisés en situation de congrès (ou d'enseignement) que dans les écrans d'EAO. Un second titre, moins communicatif certes mais plus sérieux, pourrait être «Pour une rhétorique consciente et une ergonomie raisonnée des transparents et écrans d'EAO» (titre qui logiquement devrait être imprimé en blanc sur fond bleu Méditerranée...).

2 En tout cas, ces deux titres s'appuient sur le postulat de la sémiotique qu'il existe des lois générales de la signification et de la communication, lois que l'on ne peut transgresser, en matière de communication visuelle, sous peine d'engendrer illisibilité, et donc incompréhension et rejet par le récepteur.

3 Menant une recherche action-formation pour le CNRS depuis une douzaine d'années sur la présentation des communications scientifiques en anglais dans les congrès internationaux, j'ai assisté en observateur à un certain nombre de congrès scientifiques $\mathrm{y}$ ai été frappé par le très mauvais niveau de communication, souvent dû à des performances linguistiques médiocres de la part des non-anglophones mais surtout résultat de cette croyance naïve que le contenu scientifique garantit automatiquement une bonne réception-compréhension puisque l'on s'adresse à ses pairs. Cette attitude se traduit par une non-prise en compte des facteurs qui entrent dans la réception, ou qui globalement, font que l'essentiel du message est compris. Pour dire les choses simplement et limiter l'étendue de ce propos à la communication, bien peu nombreux sont les chercheurs qui se disent qu'une manière de compenser leur anglais médiocre serait 
d'assurer la réception du contenu au moins par le biais de leurs transparents (ou supports visuels quels qu'ils soient). Il est possible d'ailleurs aussi que certains ne se le disent que trop. Pour assurer on va donc tout écrire, tout montrer. Le résultat est connu de tous :

- transparents surchargés d'information, véritables pages de textes, lues par le conférencier et souvent préfacées d'un “je sais que mon transparent est mauvais mais... (je vous le montre quand même)", ou de son équivalent.

- codage visuel aléatoire ou incohérent, rarement au service de la réception de l'information.

- la plupart du temps utilisation de la couleur pour faire joli et enluminer le rébarbatif, non pour favoriser communication ou apprentissage.

4 Les Français (faute d'être formés) ressortent à cet égard comme particulièrement mauvais aux yeux de la communauté scientifique internationale, anglo-saxonne en tout cas. En ce qui concerne l'EAO, il est facile de vérifier que l'ignorance par les concepteursmédiatiseurs des règles fondamentales de la lisibilité, de la structuration et du codage des écrans, est cause pour l'apprenant d'erreurs de lecture et d'échec dans l'exécution des tâches qui lui sont demandées.

5 Il m'a donc semblé utile de faire une recherche en la matière et de formuler à partir de là une série de règles simples de la communication visuelle pouvant être transmises et pratiquées au sein du cours de langue de spécialité, règles naturellement mises en pratique par l'enseignant lui-même ou par l'auteur-concepteur de didacticiels.

6 La recherche entreprise a rapidement fait apparaître la nécessité d'acquérir une connaissance minimale de la perception visuelle, de la lisibilité typographique, de la dimension physiologico-psychologique des couleurs, ajoutée à leur valeur symbolique et des spécificités de chaque type de support visuel (page imprimée. transparent, diapositive, poster, vidéo, écran d'ordinateur). Faute de place, seuls les trois premiers points seront abordés ici.

\section{La perception visuelle}

7 Dans le processus de lecture, l'œil parcourt la ligne par avances saccadées à grande vitesse durant lesquelles toute vision est impossible, ponctuées de pauses de fixation/ perception, d'une durée de l'ordre de 200 à 250 millisecondes. Parfois l'œil revient en arrière (régression), sur ordre du cerveau, pour réexaminer ce qui n'a pas été clairement $\mathrm{vu}$, perçu ou compris. Plus des neuf dixièmes du temps de lecture sont ainsi consacrés aux pauses de fixation et de régression. Le temps nécessaire à la lecture peut donc être considérablement allongé par effet cumulatif de facteurs de ralentissement tels que l'emploi d'une typographie dont la lisibilité est médiocre, ou des pages de textes surchargées ou un contenu trop dense.

8 Dans le processus de lecture, chaque mot est reconnu globalement (et non pas comme addition de lettres séparées - Cattell 1885). C'est la longueur et la forme caractéristique du mot qui en permettent la reconnaissance (Erdmann \& Dodge 1898). Ce sont les jambages des consonnes qui contribuent le plus à donner à un mot sa forme caractéristique (Goldscheider - Muller 1893).

9 En conséquence, on n'écrira jamais en majuscules sauf pour mettre en relief un mot isolé, un nom propre ou énoncer par exemple, un symbole chimique ou un sigle.

10 La perception de la page est globale avec, pour les civilisations ou l'on écrit de gauche à droite, le trajet suivant indiqué à la figure 1. 
Figure 1
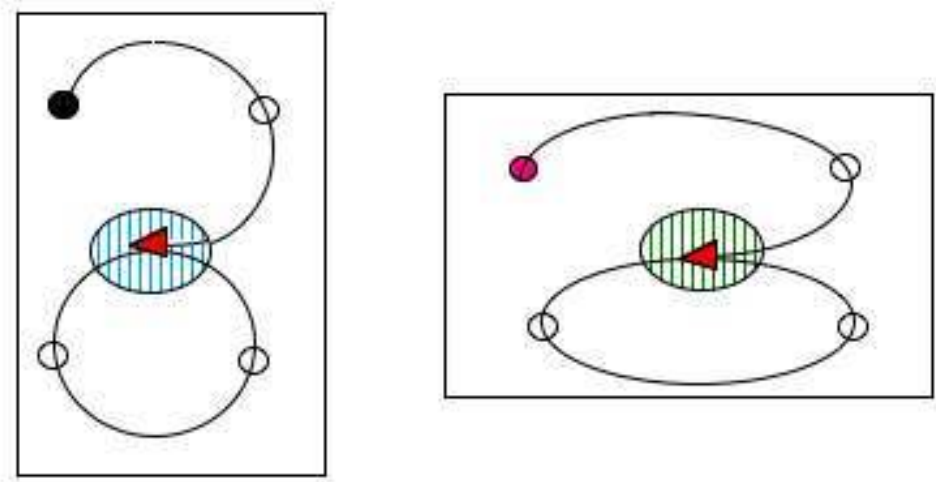

11 L'œil se fixe d'abord en haut à gauche puis parcourt la page en un mouvement incomplet, en passant deux fois par le centre pour y arrêter en fin de course.

En conséquence, le point en haut et à gauche est stratégiquement important. C'est là que l'on mettra le titre du transparent de la diapo ou de l'écran, avec en plus petit et audessus le titre (en raccourci si nécessaire) de la communication ou, encore si besoin est, de la tête de chapitre (running title). Le titre sera donc toujours décentré.

13 Les endroits où l'œil prend un virage prononcé sont des points de ralentissement et, de ce fait, également d'importance stratégique, moindre cependant que le premier (cf. paragraphe précédent). On placera donc le numéro du transparent en haut et à droite, et éventuellement le logo du labo, le titre du congrès (d'importance secondaire) en bas.

L'œil passe deux fois et s'arrête à la seconde fois au centre de la page. C'est là que l'on placera l'information la plus importante et dont on veut que l'auditoire se souvienne.

\section{Lisibilité et typographie}

Il faut que la lisibilité soit bonne pour les personnes qui se trouvent au fond de la salle. En conséquence, la taille de caractère utilisée sera fonction du type de support, lui-même fonction des conditions de présentation (une page imprimée se lit à $40 \mathrm{~cm}$, un écran d'ordinateur à $60 \mathrm{~cm}$, un transparent ou une diapo de 5 à $25 \mathrm{~m}$, etc.). On considère aussi que la taille minimum des caractères pour un transparent doit être de 36 points pour le titre, 24 points pour les sous-titres et 18 points pour le texte. On imprimera tout en caractères gras pour assurer la meilleure lisibilité possible.

On utilisera des caractères sans serif (c'est-à-dire simples et sans ornements) de type Helvetica pour tous les supports visuels. Helvetica est de toutes les polices celle qui garantit la meilleure lisibilité. À la rigueur, Times (plus élégant, pour texte imprimé ou titres) sans jamais utiliser plus de deux polices de caractères différentes dans le même document. Ne jamais utiliser de polices d'écran, caractérisées par un nom de ville (ex. New York ou Chicago) pour imprimer. Le résultat est désastreux. Helvetica et Times sont des polices d'impression.

On évitera les italiques (dont la lisibilité est médiocre) sauf, à la rigueur, pour un mot isolé. Par ailleurs, les italiques engendrent statistiquement des réactions de rejet chez le lecteur. 
La mise en page des transparents respectera l'espacement normal entre les mots (largeur d'un e), évitera la justification à droite (car elle entraîne le non-respect du principe cidessus), évitera le centrage d'informations textuelles sur l'axe vertical de la page, limitera le volume d'information à 7 lignes par page, 10 à 12 mots par ligne (soit 60 à 70 caractères maximum), utilisera le format horizontal (paysage) pour de multiples raisons : visibilité optimale, empan de l'œil, limitation automatique du nombre de lignes, distorsion réduite à la projection, etc. En résumé, le transparent dans une ergonomie optimale pourrait se présenter comme celui de la figure 2.

Figure 2

Running title $14-18$ points

\section{OHP TITLE - 36 points}

Subtitles $\quad-24$ points

- Text $\quad-18$ points

- 10-12 W/line (60-70 ch)

- $\max 240-280$ characters

- about 7 lines

- Helvetica bold

- Lower case

- Power Point (3.0) or Persuasion

La mise en page des écrans d'EAO doit respecter tous les principes énoncés plus haut en réduisant encore le volume d'informations affiché. Il est recommandé en effet de ne pas dépasser par écran 7 lignes de 40 caractères ou 5 lignes de 50 caractères. Au-delà de $40 \%$ de l'occupation totale de l'écran (soit 700 caractères sur 1 possibles), l'écran est surchargé.

La mise en page doit être systématique et ne jamais varier au sein d'un même didacticiel (voire d'une même famille de didacticiels) en matière d'ergonomie visuelle. En d'autres termes, le bandeau d'aide fonctions ou menu (qu'on situera en général en bas ou en haut de l'écran) ne doit jamais changer de place. Il en va de même pour les zones d'affichage d'instructions, de réponses et de commentaires qui auront fait l'objet d'une étude ergonomique raisonnée, fondée sur les principes de la perception de la page énoncés plus haut, ainsi que sur la nécessité d'un chemin de lecture logique et clairement structuré, condition sine qua non à la compréhension élémentaire du mode opératoire par l'apprenant. Un écran structuré de manière claire, logique et rigoureuse permet de réduire le risque d'erreur de $60 \%$ et de réduire d'un tiers le temps passé sur une tâche spécifique. 


\section{Les codages didactiques}

21 toute particulière en fonction de l'objectif recherché (rhétorique consciente).

\subsection{Mettre en relief (un mot, un titre, une information)} peut être utilisée en aveugle, simplement pour faire joli. La couleur possède pour chacun tout à la fois une dimension physiologique, psychologique et symbolique. L'interaction de ces facteurs est, évidemment extrêmement complexe, et demande réflexion par rapport aux objectifs à atteindre. 


\subsection{La dimension physiologique} possible d'entreprendre ici un exposé, même minimal, de la valeur symbolique de chaque couleur. Disons simplement et très schématiquement, que le conférencier aura intérêt, pour assurer une communication visuelle immédiate (ne serait-ce qu'à un niveau inconscient) à s'appuyer sur le code culturel généralement associé à chaque couleur. Ainsi, comme nous le savons tous (dans les pays occidentaux au moins), rouge égale stop, danger, risque de mort, etc., et, par extension de sens, interdit et important. C'est donc une couleur à utiliser en pleine connaissance de cause dans une présentation scientifique. Vert égale "go ", départ, début, jeunesse, espoir ou permission. Le jaune, associé au soleil, est souvent symbole de lumière et donc de connaissance. À ce titre, le jaune conviendra particulièrement bien pour la mise en valeur des conclusions auxquelles on est parvenu. Il s'agit donc pour le chercheur, l'enseignant ou le médiatiseur, de mettre en œuvre véritablement une rhétorique simple mais consciente du signe visuel, sans jamais perdre 
de vue la physiologie de la vision. De ce fait, on s'attachera à respecter deux grands principes directeurs, être toujours pertinent et cohérent dans son utilisation des codes visuels, viser à une lisibilité maximale pour le récepteur, tant physiologique que sémantique.

Pour le récepteur tout deviendra alors transparent, en illustration de ce que disait déjà Swift, il y a fort longtemps : «Vision is the art of seeing things invisible ».

\section{BIBLIOGRAPHIE}

Groupe Mu. 1992. Traité du signe visuel. Paris : Seuil.

Reynolds, L. et D. Simmonds. 1984. Presentation of Data in Science. Dordrecht : Nijhoff.

Pastoureau, M. 1992. Dictionnaire des couleurs de notre temps. Paris : Bonneton, Séguret.

Gueye, M. et C. Argoud. 1989. TILT ou les codages visuels de l'information sur l'écran. Paris : Les

Éditions d'Organisation.

\section{ANNEXES}

\section{Visualisation de la couleur par une formule}

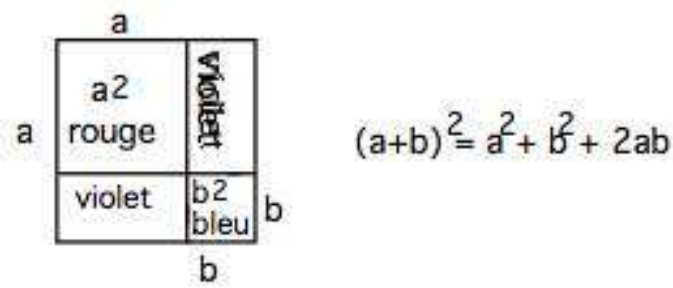

\section{NOTES}

1. La théorie des couleurs ici n'est ni physique, ni psychologique, mais le système empirique et manipulatoire nommé technologie du pigment, enseigné à l'école est connu de tous (Groupe Mu 1992 : 230, 235). 


\section{RÉSUMÉS}

Cette communication, partant d'un constat de mauvaise qualité communicative des supports visuels utilises par les chercheurs pour présenter leurs travaux scientifiques dans les congrès internationaux se fonde sur les principes de base qui régissent la physiologie de la perception visuelle et le processus de lecture, pour formuler quelques règles simples en matière de lisibilité typographique et d'ergonomie des supports visuels. Il s'y ajoute enfin quelques remarques sur les dimensions physiologiques, psychologiques et symboliques de la couleur pour essayer d'inciter les communicants à une rhétorique consciente du signe visuel. Les recommandations qui sont faites sont naturellement applicables à la médiatisation des didacticiels en EAO, tout comme à la conception de transparents d'enseignement.

The poor communicative quality of the visual aids used by researchers when presenting their work at international conferences was the starting point for the reformulation of simple rules for good "readability" based on visual perception and how one reads. The physiological, psychological and symbolic use of colour also plays a role in visual communication. The recommendations presented here are applicable to computer teaching material as well as for transparencies for the overhead projector.

\section{INDEX}

Mots-clés : communication scientifique, lisibilité, visuel

Keywords : readability, scientific communication, visual

\section{AUTEUR}

\section{ALAIN GINET}

Alain Ginet enseigne à l'Université Stendhal - Grenoble 3. 